\section{The management of anterior tooth wear using gold palatal veneers in canine guidance}

\author{
S. Eliyas ${ }^{* 1}$ and N. Martin ${ }^{2}$
}

VERIFIABLE CPD PAPER

IN BRIEF
- Stresses that the restoration of worn
maxillary anterior teeth, requiring an
increase in occlusal vertical dimension,
should aim to re-establish an occlusal
relationship in centric relation with
coincidence of retruded contact position
and intercuspal occlusal position.
Suggests establishment of a mutually
protected occlusion and restoration of
canine guidance is an effective way of
managing tooth wear.

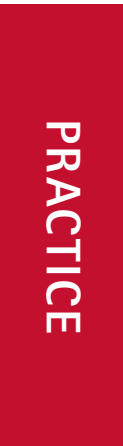

Localised anterior tooth wear can be managed using minimally invasive techniques with conservation of tooth structure and preservation of pulp vitality. This article describes and illustrates with two clinical cases, the management of localised tooth wear, with the restoration of canine guidance by a combination of gold palatal veneers and direct composite restorations.

\section{INTRODUCTION}

The prevalence of tooth wear in the UK is increasing, with a reported incidence in the adult population of $76 \%$ in 2009 compared to $66 \%$ in $1998 .{ }^{1,2}$ The severity of the tooth wear is also increasing with an incidence in the same report of 15\% classified as moderate and 2\% severe. Tooth erosion is one form of tooth wear that is increasingly affecting the younger population, causing gradual but irreversible loss of tooth structure. It affects primarily the adolescent population worldwide with a reported international incidence of up to $20 \%$ of the population and greater national incidences in specific regions. ${ }^{3}$ Given the young age of the affected population and the need for a conservative approach, it is sensible to provide a minimally invasive treatment approach. The focus of this should be to maintain as much of the dental hard tissues as possible, as it can be expected that the restorations will need refurbishing/replacing a number of times over the course of the patient's lifetime.

Historically, among British dental practitioners, there has been a perception that the management of tooth wear lies outside the scope of general dental practice and is

'Department of Restorative Dentistry, Charles Clifford Dental Hospital, Sheffield Teaching Hospitals NHS Trust, Wellesley Road, Sheffield, S10 2SZ; ${ }^{2}$ Academic Unit of Restorative Dentistry, School of Clinical Dentistry, The University of Sheffield, Sheffield, S10 2TA

*Correspondence to: Shiyana Eliyas Email: shiyanaeliyas@hotmail.com

\section{Refereed Paper}

Accepted 8 November 2012

DOI: 10.1038/sj.bdj.2013.273

${ }^{\circledR}$ British Dental Journal 2013; 214: 291-297
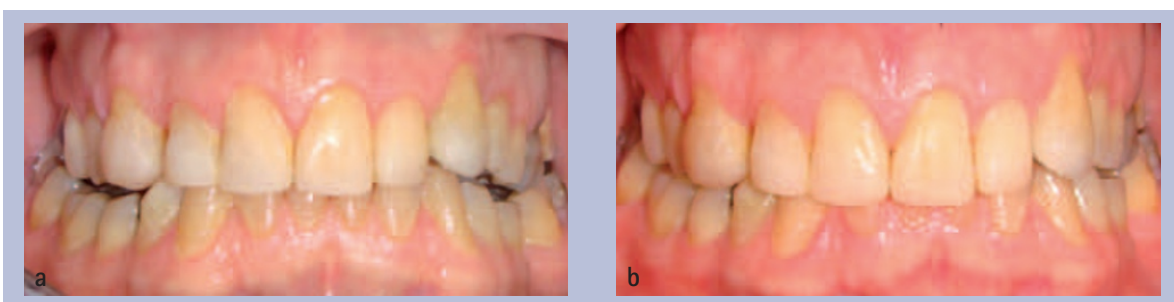

Fig. 1 The Dahl concept using composite resin and a gold palatal veneer on the UL3: (a) is immediately post fitting of a gold palatal veneer to the UL3 and composite build up of the UR3, UR2, UR1, UL1, UL2 and labial aspect of the UL3; (b) shows the change within four months

predominantly the remit of specialist care. Changes in society, principally increased longevity with tooth retention, increased patient expectations for a functional and aesthetic dentition coupled with a reduced capacity from specialist centres, has placed an increased demand on the primary care sector to provide the required restorative care to manage these cases of tooth wear. Recognising the need for a shift in care provision, a series of articles ${ }^{4-7}$ dealing with the aetiology, diagnosis and treatment of tooth wear have been widely publicised over the past two decades and this article will consider some key points.

The restorative management of the worn dentition involves working with a conformative or a reorganised approach. Conformative treatment is the restoration of the affected teeth without changing the occlusal vertical dimension (OVD) and conforming to the intercuspal occlusal (ICP) relationship that the patient presents with. A reorganised approach requires the restoration of worn teeth in centric relation (CR) with an increase in OVD. The restoration of canine guidance is an appropriate strategy in a reorganised approach, with the possible
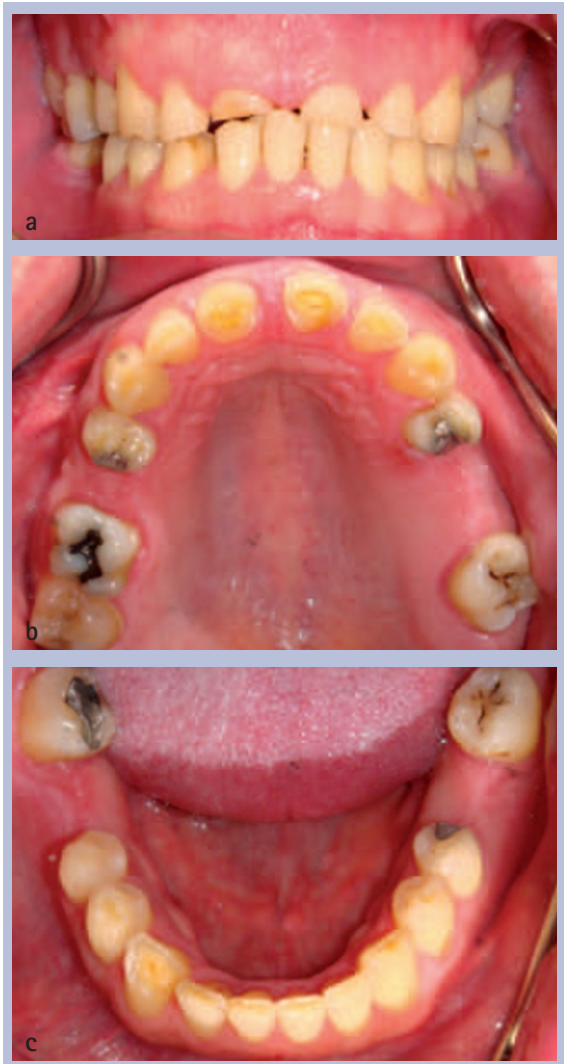

Fig. 2 Case one pre-treatment: (a) labial retracted view pre-treatment; (b) upper occlusal view pre-treatment; (c) lower occlusal view pre-treatment 
exception of cases where a large removable partial denture is involved as the security of the prosthesis may be compromised by the loss of a balanced articulation.

It is pertinent to this article to highlight the significance of the OVD in the management of tooth wear and the role of the Dahl concept in this respect. The occlusal vertical dimension is the distance measured between two points when the occluding members (teeth) are in contact. ${ }^{8}$ The increase in OVD can be as large as 20-25 $\mathrm{mm}$ (as measured between the upper and lower anterior teeth) in dentate patients as this is the space created anteriorly when the condyles are purely rotating about the condylar axis and therefore when the centric relation (the position of the mandible when the Frankfort plane is horizontal and the condyles are in the most superior, posterior, unstrained position in the glenoid fossae) remains largely unchanged.9-11 The Dahl concept is recognised as the underlying principle that should be adopted for the treatment of localised tooth wear where possible to create a temporary increase in OVD. In 1975 Dahl and Krogstad showed that a localised 'partial bite raising appliance' on upper teeth can be used to create localised interocclusal space due to a combination intrusion (40\%) of the teeth in contact with the 'Dahl' appliance and extrusion $(60 \%)$ of the teeth rendered free of contact. ${ }^{12-16}$ In more recent times the "bite raising appliances' have been replaced with composite resin restorations (Fig. 1) with success rates of $89 \%$ at thirty months. ${ }^{16}$ The Dahl concept is successful in creating space $96 \%$ of the time with no pulpal symptoms, periodontal symptoms and temporomandibular joint dysfunction in the majority of cases. ${ }^{15,16}$ As the stability of the OVD is determined by the facial musculature, ${ }^{15,17,18}$ an increase in OVD using the Dahl concept returns to that determined by the musculature intrusion of the 'built-up' teeth and extrusion of the other teeth. Dento-alveolar compensation with a loss of restorative space is often seen in tooth wear cases, however, the loss of OVD is only present if the tooth wear progresses at a rate that exceeds the rate of dento-alveolar growth and therefore compensation. ${ }^{19}$

With consideration to the restorative management of the worn dentition in
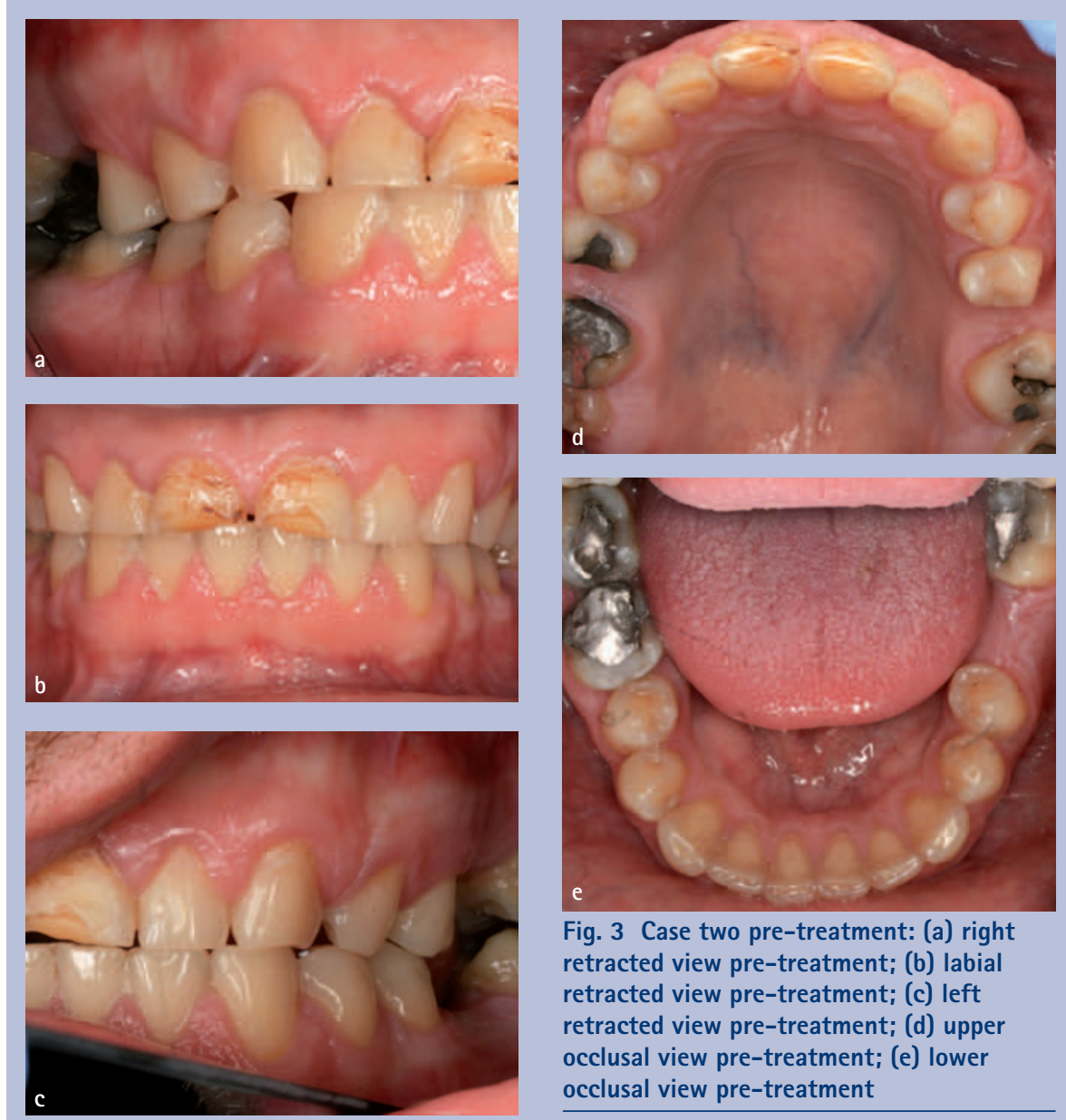

Fig. 3 Case two pre-treatment: (a) right retracted view pre-treatment; (b) labial retracted view pre-treatment; (c) left retracted view pre-treatment; (d) upper occlusal view pre-treatment; (e) lower occlusal view pre-treatment

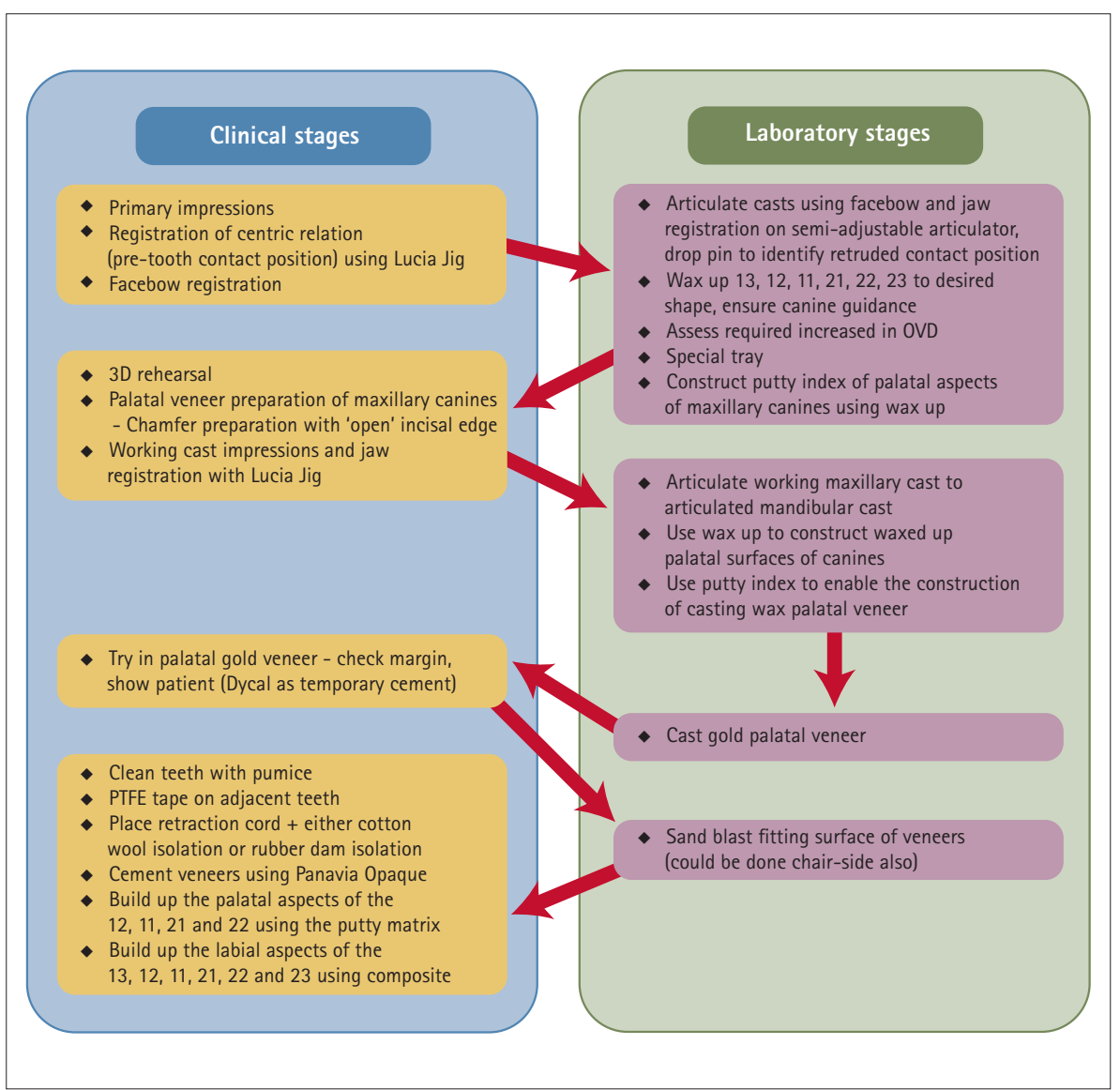

Fig. 4 Clinical and laboratory stages for this technique 


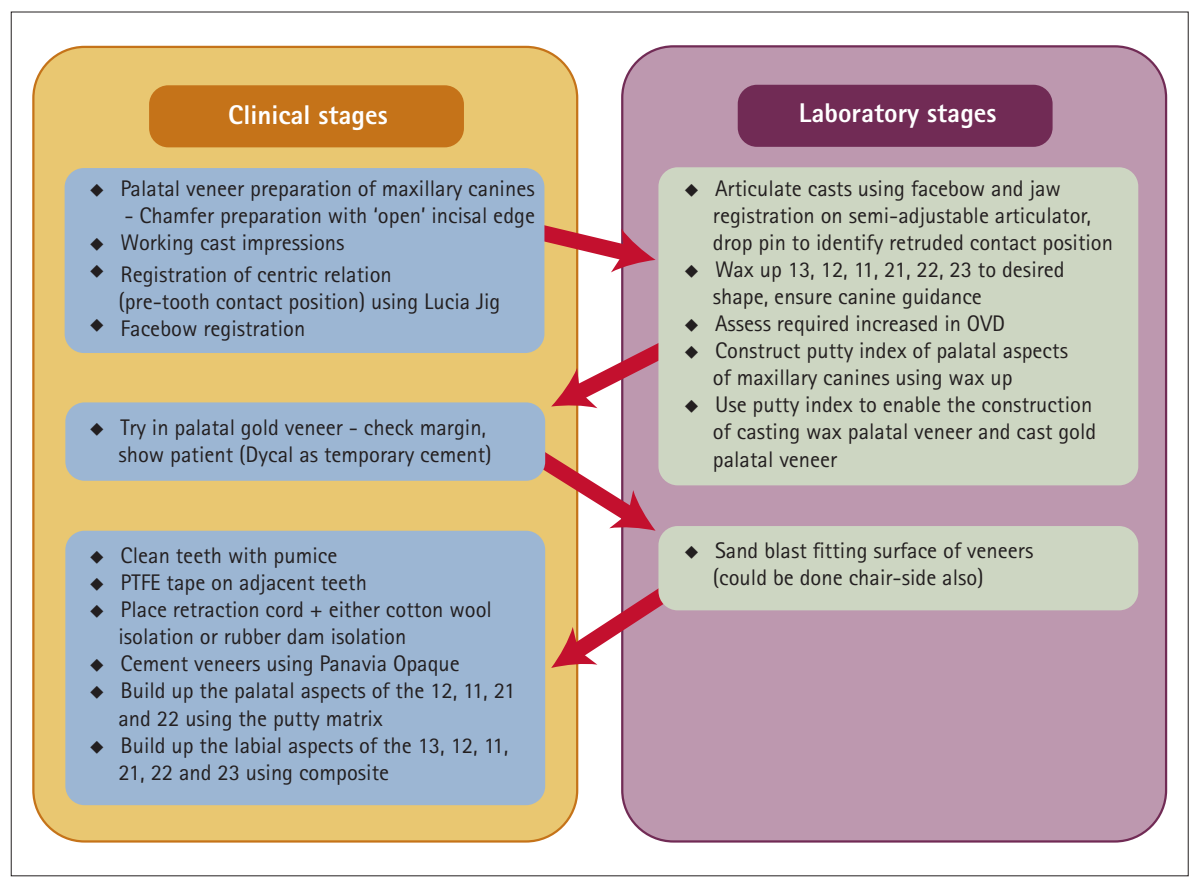

Fig. 5 Minimum clinical and laboratory stages for this technique

the dentate patient, a mutually protective occlusion or canine protected occlusion is an appropriate and often desirable option to be considered. Anterior teeth are involved in protrusion and lateral guidance while the posterior teeth disocclude. The anterior teeth come into contact before the posterior teeth to limit the loading applied, therefore reducing wear of the posterior teeth. The posterior teeth maintain stable occlusal vertical dimensions preventing wear of the anterior teeth. ${ }^{20,21}$ It is desirable to consider canine protected occlusions when restorations are used to determine lateral guidance because it is relatively easy to create compared to guidance on a number of posterior teeth on the working side (group function) and does ensure that working and non-working side interferences are not present in lateral excursions. The canine tooth is the cornerstone of the arch and in canine guidance, only the palatal surface of the maxillary canine on the working side is in contact, with disocclusion of all the posterior teeth in the (contra-lateral) non-working side. The appropriateness of this tooth for guiding the occlusion is highlighted by its favourable crown to root ratio being suitable for absorbing occlusal forces; the root of the canines is longer and has a greater surface area providing more periodontal ligament and periodontal proprioreceptors in comparison to the adjacent teeth; the concave palatal aspect is highly suited to gliding lateral movements; its ideal position in the mouth to resist levering forces; and it's the tooth which remains longest in the mouth. ${ }^{10}$ There is, however, no conclusive evidence for canine protected occlusion being superior to any other type of occlusion. ${ }^{10,21}$

While the lost tooth surface of anterior teeth can be restored with direct and indirect techniques with great success as reported in various studies; $;, 22-30$ the use of direct-placement composite restorations is considered a highly conservative approach. ${ }^{25}$ Although the ten-year cumulative survival estimates of resinbased composites have been reported to be lower than ceramo-metal crowns (62\% compared with 74.5\%), the failures have been due to fracture and deemed easier to replace or repair compared to root canal treatment or extraction of the failed ceramo-metal crowns. ${ }^{26}$ The provision of direct, minimally invasive restorations does not limit the use of the other restorations in the future and means that the lifetime of the more destructive restoration starts later.

Tooth wear can be multi-factorial in its aetiology, thus, while direct-placement composite restorations provide a highly conservative restorative modality, the increased 'wear' burden on guiding canines compared to other teeth may lead to premature wear or fracture of these restorations, compromising the longevity of

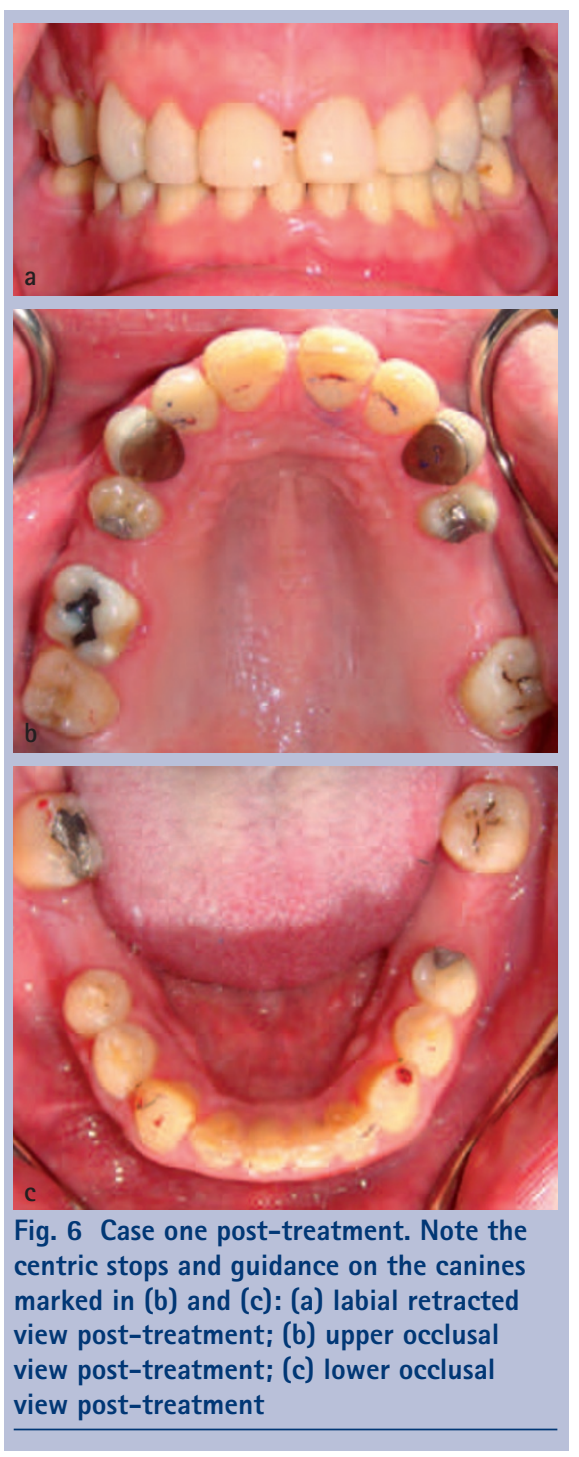

the composite restorations on other worn anterior teeth. This is especially so in those with parafunctional habits. An alternative strategy using cast metal restorations for the guiding palatal surface has been advocated and despite the increased initial cost, it can reduce time required in the dental chair and result in a more predictable and durable solution due to the higher wear resistance. ${ }^{5,31}$

The aim of the treatment strategy outlined in this article is to adhere to the conservative principles of minimum intervention, balanced with durability of canine guiding surfaces. This is achieved by combining direct-placement composite restorations and gold-palatal veneers on canine surfaces. This technique with stepby-step clinical and technical protocols is detailed and further illustrated with two clinical case reports that use the Dahl concept of dento-alveolar compensation to re-establish posterior support. 


\section{PRACTICE}
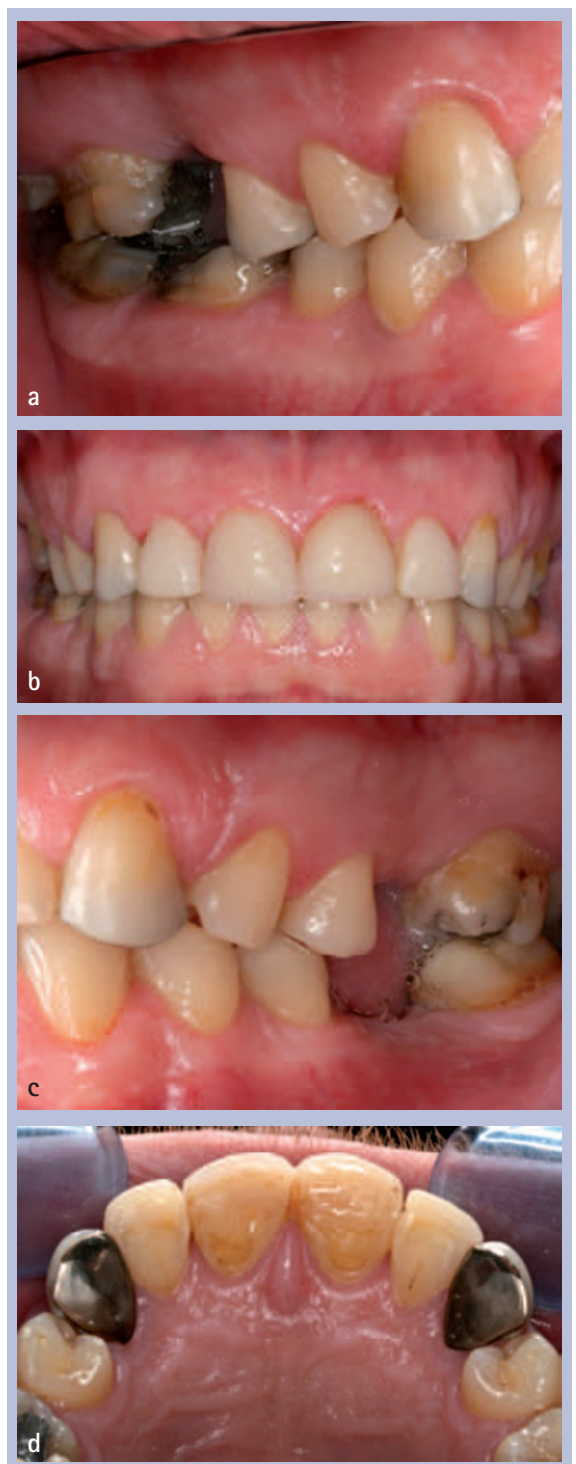

Fig. 7 Case two post-treatment: (a) right lateral view post-treatment; (b) labial retracted view post-treatment; (c) left lateral view post-treatment; (d) upper lateral view post-treatment

\section{CLINICAL CASES}

The following two patients were in their mid-thirties and presented with tooth wear localised mainly to the upper anterior teeth. Their complaints related to their appearance and both presented with adequate oral hygiene and good periodontal support. There had been a loss of restorative space as a result of the tooth wear. A treatment strategy with a conservative approach was sought.

In case one, the tooth wear was localised but also affected the mandibular right canine; this was predominantly erosive in nature (associated with a historic intake of soft drinks as the main thirst-quenching drinks) with an element of attrition (Fig. 2). In case two, the maxillary central incisors
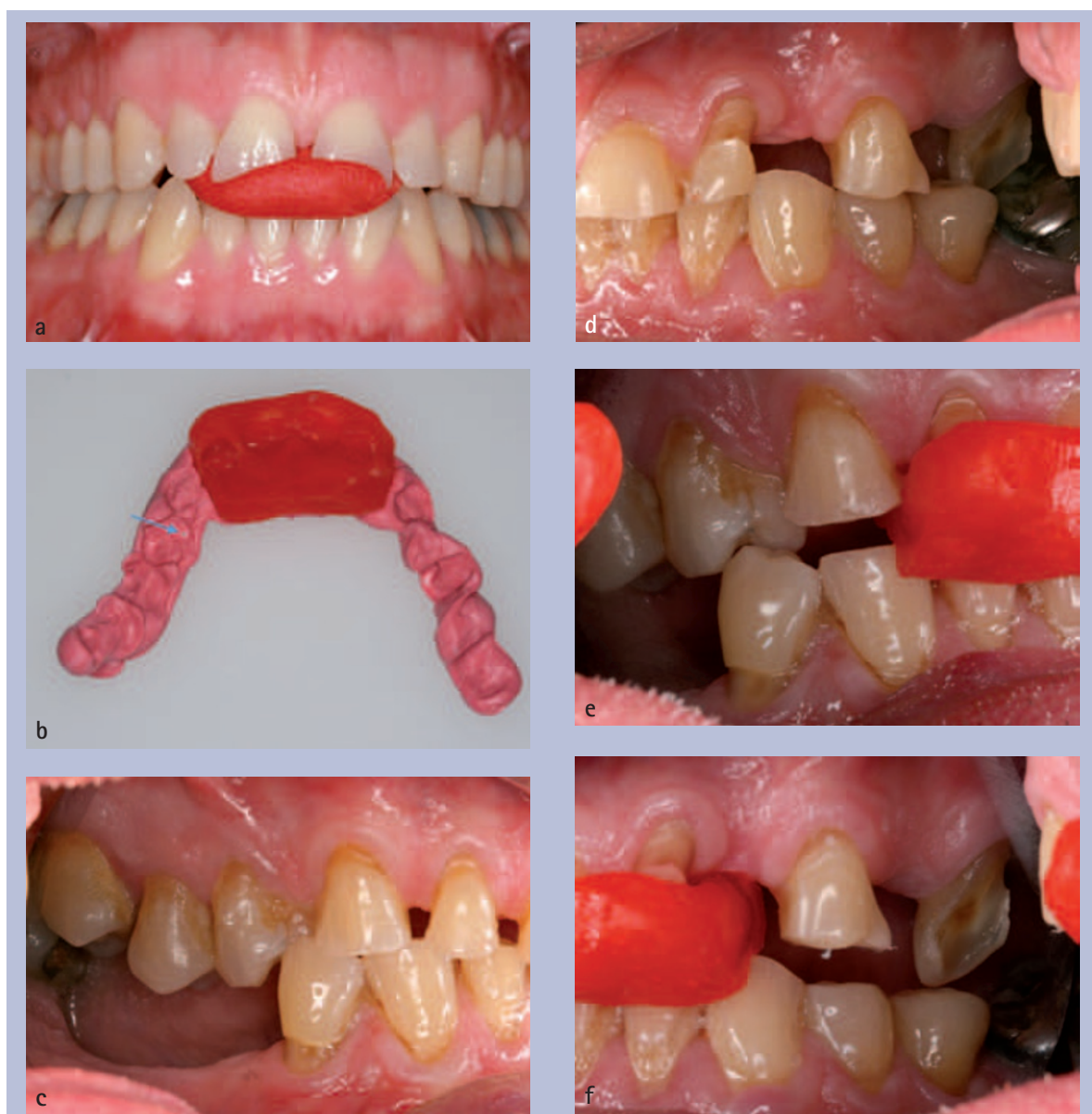

Fig. 8 (a) Labial retracted view of Duralay acrylic Lucia Jig; (b) an interocclusal recording material has been used to record the posterior teeth in (b) and the blue arrow shows the contact recorded in the registration and demonstrates the effective recording of CRCP; (c) right lateral view without Lucia Jig in situ; (d) left lateral view without Lucia Jig in situ; (e) right lateral view with Lucia jig in situ; (f) left lateral view with Lucia Jig in situ

had previously been restored with direct placement composites to protect the incisal edges. The tooth wear was predominantly due to parafunctional attrition (bruxism) with an erosive component associated with regular drinking of soft-drinks (Fig. 3).

The aim of treatment in both cases was the restoration of function and aesthetics with a conservative management of the maxillary and mandibular anterior sextants using direct-placement composites to allow dento-alveolar compensation to re-establish posterior contacts. A canineguided mutually protected occlusion was designed to protect the anterior composite restorations. This aim was achieved in the following sequence of stages:

1. Establishment of the pre-tooth contact position or centric relation (CR) using a Lucia jig $^{32}$ and recording this using a Lucia jig and hard base plate wax

2. Creating an increased OVD of 5-6 mm to enable the restoration of the anterior sextants to full anatomical height and form. The desired occlusal relationship was designed and waxed-up on a semi-adjustable articulator using the $\mathrm{CR}$ record obtained in clinic

3. Provision of gold veneers on the palatal guiding surfaces of the canines, as per the diagnostic wax-up. A step-by-step guide to the technical, clinical stages and the integration of these is detailed in Figures 4 and 5

4. Restoration of the maxillary and mandibular anterior sextants with direct placement composite using a siliconeputty index from a diagnostic wax-up.

The desired outcome was achieved having created a conservative, maintainable, functional and aesthetic outcome. Posterior dento-alveolar compensation occurred within 18 weeks with reestablishment of full occlusal contact. A mutually protected occlusal scheme was achieved with canine guidance, protrusive guidance shared on the maxillary anterior 
sextant and posterior disocclusion (case one - Fig. 6; case two - Fig. 7).

\section{OPERATIVE CONSIDERATIONS TO ACHIEVING AN OPTIMAL RESULT}

\section{Centric relation and increase in OVD}

Primary impressions, facebow recording and the use of a Lucia jig are essential to record the CR occlusal relationship (Fig. 8). It may be possible to achieve restorative space by manipulation of the mandible to the retruded contact position (RCP), where the first tooth contacts in the $\mathrm{CR}$ arc of closure, in patients who have a slide from RCP to ICP. Where the $\mathrm{RCP}$ and ICP are coincident, CR must be recorded and OVD increased to ascertain restorative space. The desired increase in OVD can be identified and recorded using a Lucia jig made of DuraLay (Reliance Dental Manufacturing Co., Worth, Illinois, USA) and a rigid registration wax (for example, Moyco Beauty Pink-X-Hard, Moyco Industries Inc., Philadelphia, USA) or a wax rim where some anterior teeth are missing or are severely worn. DuraLay acrylic is very dimensionally stable and can be used multiple times during treatment; however, it can cause discomfort from polymerisation heat and is more expensive than Green Stick Composition (Kerr Manufacturing Co. Romulus, Michigan, USA), which is easy to use but dimensionally unstable and therefore cannot be used on multiple occasions due to distortion and fracture. Before using either material, the teeth should be coated with petroleum jelly to prevent the material adhering to the tooth.

This can be achieved either at the chairside as a clinical procedure or on the articulated casts. The clinical technique requires an element of experience and by its very nature the clinician has direct control over the desired outcome. The technical process requires that the casts are articulated on a semi-adjustable articulator using a facebow and CR record and subsequently the pin can be lowered to identify the required increase in occlusal vertical dimension for the ideal wax-up of the teeth. An example is shown in Figure 9.

\section{Three-dimensional rehearsal or 'mock-up'}

A vacuum-formed mould or a putty index of the wax up could be used to carry out a 'mock-up' using a provisional composite material to show the patient the proposed treatment and desired appearance. The putty index can be used later on for the composite 'build-ups' also.

\section{Fitting the gold palatal veneers}

The teeth to be restored need to be carefully stripped of any previous restorations to maintain the maximum surface area of enamel for bonding.

For the palatal gold veneer, minimal preparation of the palatal aspect of the canine is recommended to establish a finish line and continuity of the veneertooth surfaces (Fig. 10). The incisal edge requires no preparation at the initial veneer preparation stage. A balance has to be reached between preservation of tooth structure and predictability/durability of the restored tooth complex. The authors feel that the minimum preparation required for the veneers together with the increased wear resistance of the gold tips the balance in favour of the this technique. It is appreciated that there is a biological cost involved in preparation for gold palatal veneers that may be avoided using direct composite restorations and putty matrices or transparent custom stents can be used to ensure minimal reduction of tooth structure. ${ }^{33}$ The use retraction cord is advisable to record an effective impression of the margin.

The maxillary working cast is articulated used the Lucia jig and the wax wafer taken following tooth preparation to the primary lower cast previously articulated using the facebow registration in the laboratory. Using the putty matrix of the initial wax up the laboratory can construct palatal veneers with ideal canine guidance. The lateral guidance must be designed so that it lies purely on the gold palatal veneer and not at the margin between the gold and composite to prevent shearing forces (which may lead to fracture or peeling of the composite off the gold surface).

The palatal veneers can be tried in the mouth using Dycal ${ }^{\circledR}$ (Dentsply Ltd, Addlestone, Surrey, UK) as described by Poyser et al. $^{34}$ Once the gold veneers are placed, a small amount of composite could be added (without etch and bond) to show the patient the appearance. If the patient is happy the composite and the veneer can
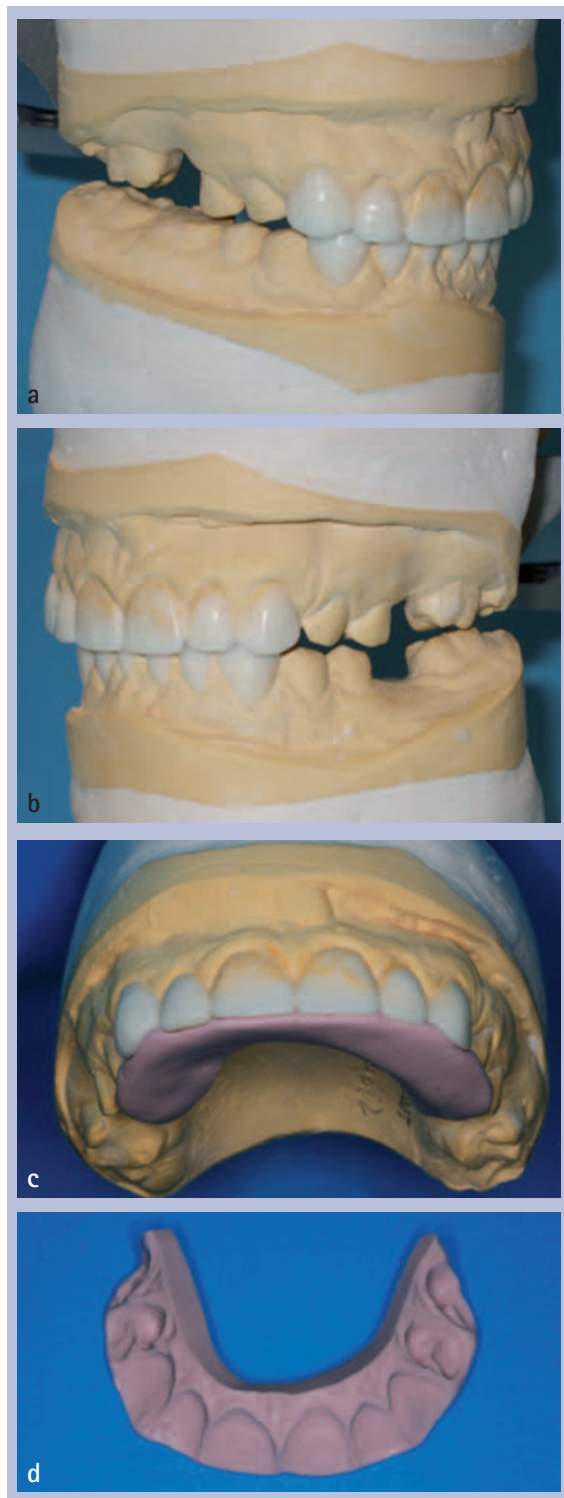

Fig. 9 Diagnostic wax-ups on semiadjustable articulators. (c) and (d) show the putty index that can be used to construct the gold palatal veneers

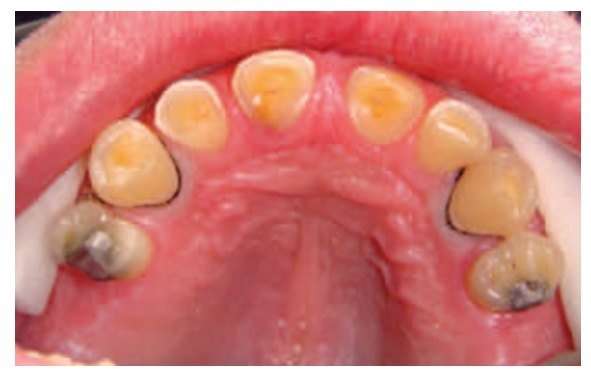

Fig. 10 Palatal gold veneer preparation and using dry retraction cord to prevent contamination of etched enamel with gingival crevicular fluid

easily be removed with a probe and light finger pressure. Once tried in the mouth the fitting surface of the veneer must be sandblasted with aluminium oxide particles, which are usually 50 microns in size before fitting. 
Before cementation of the gold palatal veneer, it is advisable to prepare the incisal edge with a long shallow chamfer or bevel to ensure an effective blending of the labial incisal composite restoration to improve the aesthetic result although no preparation of the teeth that are receiving composite is necessary to improve longevity of the composite restorations. ${ }^{25,36}$

Good moisture control during cementation is essential. Dry retraction cord is required to prevent contamination with gingival crevicular fluid and should be used for both cementation of the veneers and composite build-ups. Rubber dam is suggested as a further strategy to achieve effective isolation where possible, ensuring that the margins of the dam are everted to provide an effective seal and so that the cervical margins of the tooth preparation are visible to ensure full seating of the restorations. In the anterior maxilla, labial cotton wool rolls may be used instead of rubber dam, as moisture control is easier. The teeth should be cleaned with the careful use of slow-speed rotary soft rubber-cup and a water-based slurry of flour of pumice to avoid soft-tissue abrasion of the gingivae and subsequent bleeding that will interfere with the adhesive protocols. White PTFE tape (Poly-tetra fluoro-ethylene thread seal tape or "plumbers tape') can be used to prevent adherence of the cement to adjacent teeth (Fig. 11). The palatal veneers can be cemented with a dual-cure resin-based composite lute or, as is the authors' preference, with the opaque shade of Panavia 21 (Kuraray Co. Ltd, Kita-Ku, Osaka, Japan) following sandblasting. ${ }^{34}$ We have tried various methods of masking the gold, to reduce 'show-through' in the composite incisal tip. We found the most effective technique was to sand-blast the gold-fitting surface and use a translucent shade of Panavia 21 (Kuraray Co. Ltd, Kita-Ku, Osaka, Japan) resin lute; followed by a restorative composite of shade matching the patients natural dentition for the restoration of the incisal tip. As canines are usually 'warmer' in colour compared to the incisors the appearance is often acceptable to the patients.

\section{Composite restorations or 'build-ups'}

The teeth to be restored need to be carefully stripped of any previous restorations to maintain the maximum surface area
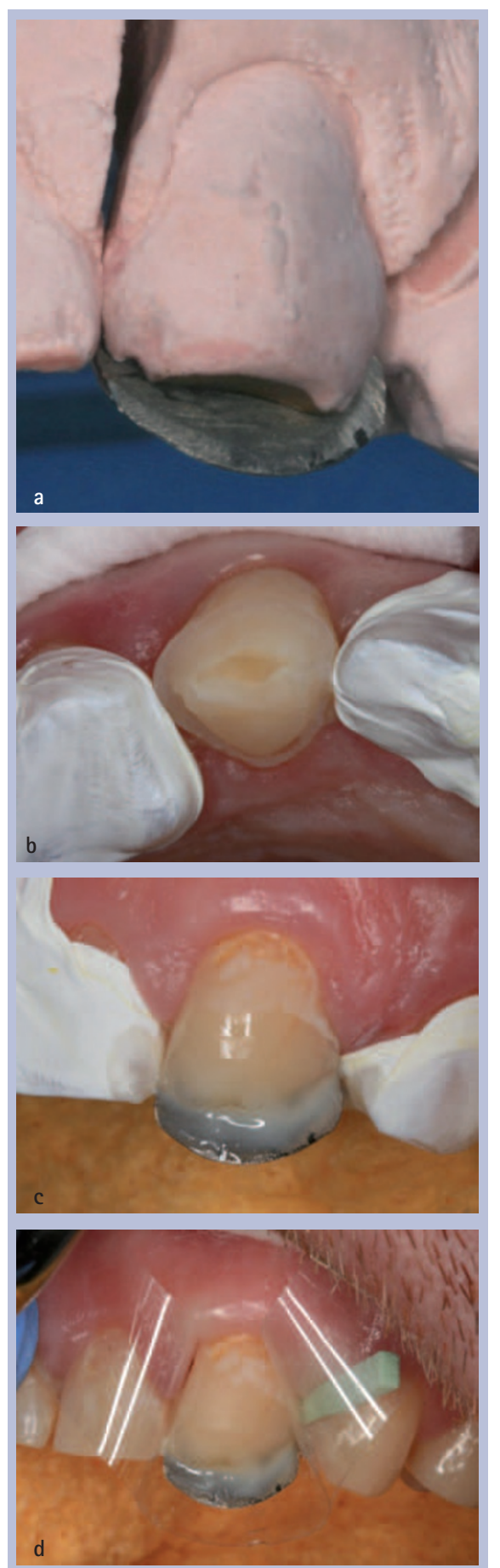

Fig. 11 Cementing gold palatal veneers: (a) gold palatal veneer on the cast; (b) tooth prepared for a gold palatal veneer with PTFE tape on the adjacent teeth to isolate the prepared tooth; (c) cementation of gold palatal veneer with PTFE tape on the adjacent teeth; (d) cementation of gold palatal veneer with celluloid strips on the adjacent teeth

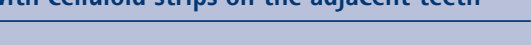

of enamel for bonding. The teeth should be cleaned with a slow-speed rotary soft rubber-cup and a water-based slurry of pumice. Again good moisture control using rubber dam or labial cotton wool rolls and retraction cord where appropriate is recommended.

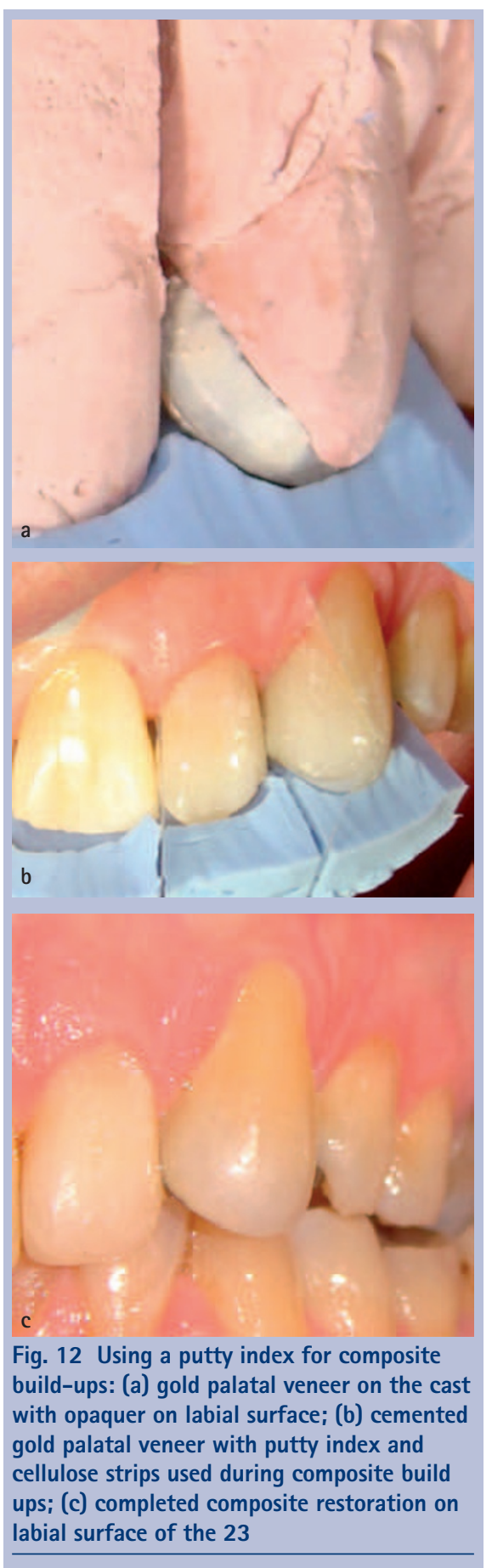

Following cementation of the palatal veneer, the putty matrix used to construct the veneer can be disinfected and used clinically to then build up the maxillary central and lateral incisors. The putty index (Fig. 12) can be sectioned through the incisal edges and used to aid building up of the palatal aspects of the lateral and central incisors following placement of white PTFE tape or cellulose strips to maintain the contact separation between the composite restorations on adjacent teeth. The putty index can also be sectioned partially interdentally to facilitate 
the placement of clear cellulose strips if this technique is preferred to PTFE tape. A circumferential bevel preparation of the incisal edges is normally advocated to achieve a more effective blend of the composite with the underlying tooth; thus achieving a more aesthetic result. The design and magnitude of this varies among clinicians and recommendations range from a minimal short bevel to a long-shallow bevel ( 2 to $4 \mathrm{~mm}$ ), a combination of the two (long labial and short palatal) or even a so-called starburst bevel. ${ }^{36-38}$

\section{CONCLUSION}

The approach detailed in this paper to managing tooth wear (especially that localised to the upper anterior teeth) is a minimally invasive technique. It is acknowledged that any form or interventive restorative treatment is subject to maintenance that may involve repairs and/or restoration replacement. The long term repair and maintenance of the gold restorations is likely to extend the longevity of the composite restorations by reducing the occlusal impact of undesirable interferences. For experienced users of the technique the stages could be reduced to that shown in Figure 5 with three-dimensional rehearsal being carried out at the initial assessment.

To summarise, the principles for the restoration of worn maxillary anterior teeth using this technique are:

- Re-establishment of an occlusal relationship in CR and therefore coincident RCP and ICP

- Change in OVD commensurate with the degree of tooth wear and appropriate to the restoration of the dentition to natural form and size

- Establishment of a mutually protected occlusion and restoration of canine guidance

- Use of the Dahl concept (dento-alveolar compensation) to re-establish occlusal contact of the posterior sextants

- Consideration given to the combined use of gold palatal veneers for the guiding canines and direct-placement composite restorations as a predictable and durable strategy for the restoration of the worn maxillary anterior sextant.

1. The NHS Information Centre. Adult Dental Health Survey 2009. London: The Health and Social Care Information Centre, 2010

2. Nunn J, Morris J, Pine C, Pitts N B, Bradnock G, Steele J. The condition of teeth in the UK in 1998 and implications for the future. Br Dent J 2000; 189: 639-644.

3. El Aidi H, Bronkhorst E M, Truin G J. A longitudinal study of tooth erosion in adolescents. J Dent Res 2008; 87: 731-735.

4. Kelleher M, Bishop K. Tooth surface loss: an overview. Br Dent J 1999; 186: 61-66.

5. Mehta S B, Banerji S, Millar B J, Suarez-Feito J M. Current concepts on the management of tooth wear: part 2. Active restorative care 1: the management of localised tooth wear. Br Dent J2012; 212: 73-82.

6. Chu F C, Yip H K, Newsome P R, Chow T W, Smales $\mathrm{R} J$. Restorative management of the worn dentition: 1. Aetiology and Diagnosis. Dent Update 2002; 29: $162-168$

7. Porter R, Poyser N, Briggs P, Kelleher M. Demolition experts: management of the parafunctional patient: 1. Diagnosis and prevention. Dent Update 2007; 34: 198-200, 202-204, 207

8. The glossary of prosthodontic terms. J Prosthet Dent 2005; 94: 10-92.

9. Posselt U. Studies in the mobility of the human mandible. Acta Odontol Scand 1952; 10: 10.

10. Pameijer J H N. Periodontal and occlusal factors in crown and bridge procedures. Amsterdam: PBC, 1985

11. Dawson P E. Functional occlusion: from TMJ to smile design. St. Louis, MO: Mosby Elsevier, 2007.

12. Dahl B L, Krogstad O, Karlsen K. An alternative treatment of cases with advanced localised attrition. J Oral Rehabil 1975; 2: 209-214.

13. Dahl B L, Krogstad O. The effect of a partial bite raising splint on the occlusal face height. An x-ray cephalometric study in human adults. Acta Odontol Scand 1982; 40: 17-24.

14. Dahl B L, Krogstad O. The effect of a partial biteraising splint on the inclination of upper and lower front teeth. Acta Odontol Scand 1983; 41: 311-314.

15. Dahl B L, Krogstad O. Long-term observations of an increased occlusal face height obtained by a combined orthodontic/prosthetic approach. J Oral Rehabil 1985; 12: 173-176.

16. Haemmings K W, Darbar U R, Vaughan S. Tooth wear treated with direct composite restorations at an increased vertical dimension: results at 30 months. J Prosthet Dent 2000; 83: 287-293.

17. Christensen J. Effect of occlusion-raising procedures on the chewing system. Dent Pract Dent Rec 1970; 20: 233-238.

18. Lindauer S J, Gay T, Rendell J. Effect of jaw opening on masticatory muscle EMG-force characteristics. J Dent Res 1993; 72: 51-55.

19. Berry D C, Poole D F. Attrition: possible mechanisms of compensation. J Oral Rehabil 1976; 3: 201-206.
20. Rinchuse D J, Kandasamy S, Sciote J. A contemporary and evidence-based view of canine protected occlusion. Am J Orthod Dentofacial Orthop 2007; 132: 90-102.

21. Shillingburg $H T$, Hobo $S$, Whitsett $L D$, Jacobi $R$, Beckeett $S$ E. Fundamentals of fixed prosthodontics. 3rd edn. Chicago: Quintessence, 1997.

22. Gough M B, Setchell D J. A retrospective study of 50 treatments using an appliance to produce localised occlusal space by relative axial tooth movement. $\mathrm{Br}$ Dent J 1999; 187: 134-139.

23. Redman C D, Haemmings K W, Good J A. The survival and clinical performance of resin-based composite restorations used to treat localised anterior tooth wear. Br Dent J 2003; 194: 566-572.

24. Chu F C, Sui A S, Newsome P R, Chow T W, Smales $\mathrm{R} J$. Restorative management of the worn dentition: 2. Localized anterior toothwear. Dent Update 2002; 29: 214-222.

25. Poyser N J, Briggs P F, Chana H S, Kelleher M G Porter R W, Patel M M. The evaluation of direct composite restorations for the worn mandibular anterior dentition - clinical performance and patient satisfaction. J Oral Rehabil 2007; 34: 361-376.

26. Smales R J, Berekally T L. Long-term survival of direct and indirect restorations placed for the treatment of advanced tooth wear. Eur J Prosthodont Restor Dent 2007; 15: 2-6.

27. Mehta S B, Banerji S, Millar B J, Suarez-Feito J M Current concepts on the management of tooth wear: part 3. Active restorative care 2: the management of generalised tooth wear. Br Dent J. 2012; 212: 121-127.

28. Mehta S B, Banerji S, Millar B J, Suarez-Feito J-M Current concepts on the management of tooth wear: part 4. An overview of the restorative techniques and dental materials commonly applied for the management of tooth wear. Br Dent J. 2012; 212: 169-127.

29. King P A. Adhesive techniques. Br Dent J 1999; 186: 321-326.

30. Setchell D J. Conventional crown and bridgework. BrDent J 1999; 187: 68-74

31. Suzuki S, Nagai E, Taira Y, Minesaki Y. In vitro wear of indirect composite restoratives. J Prosthet Dent 2002; 88: 431-436.

32. Lucia $\vee 0$. A technique for recording centric relation. J Prosthet Dent 1964: 14: 492-505.

33. Lynch C D, Burke F M. Controlled tooth reduction for fixed restorations using custom made precontoured transparent templates. J Prosthet Dent 2004; 91: 499-500.

34. Poyser N J, Briggs P F. The Dycal try-in technique for resin bonded bridges. Dent Update 2004; 31: 393-394, 396-397.

35. Baratieri L N, Ritter A V. Critical appraisal. To bevel or not in anterior composites. J Esthet Restor Dent 2005: 17: 264-269.

36. Manhart J. Aesthetic layering technique. The Dentist 2009. Online article available at http://www.voco. com/en/products/_products/amaris/AB_Amaris Dr_Manhart THE_DENTIST_March_2009.pdf (accessed March 2013).

37. LeSage B P. Aesthetic anterior composite restorations: a guide to direct placement. Dent Clin North Am 2007; 51: 359-378, viii

38. LeSage B P, Milnar F, Wohlberg J. Achieving the epitome of composite art: creating natural tooth esthetics, texture, and anatomy using appropriate preparation and layering techniques. J Cosmet Dent 2008; 24: 42-51. 